ESJ Humanities

\title{
Statut social et émigration internationale des femmes au Burkina Faso
}

\author{
Ousmane Barry
}

Université Joseph Ki-Zerbo, Burkina Faso. Institut National de la Statistique et de la Démographie (INSD), Burkina Faso

\author{
Honoré Mimche \\ Patrice Tanang Tchouala \\ Hamidou Kone
}

Institut de FOrmation et de Recherche Démographiques (IFORD),

Yaoundé, Cameroun

Doi:10.19044/esj.2021.v17n20p163

Submitted: 01 May 2021

Accepted: 18 June 2021

Published: 30 June 2021
Copyright 2021 Author(s)

Under Creative Commons BY-NC-ND

4.0 OPEN ACCESS

Cite As:

Barry O., Mimche H., Tchouala P.T. \& Kone H. (2021). Statut social et émigration internationale des femmes au Burkina Faso. European Scientific Journal, ESJ, 17(20), 163. https://doi.org/10.19044/esj.2021.v17n20p163

\section{Résumé}

Le statut social de la femme est un critère de différenciation dans le domaine de la migration internationale féminine. Cependant la littérature reste peu prolixe sur la relation entre celui-ci et la pratique migratoire. Cette étude vise à documenter les liens entre le statut des femmes et leurs comportements migratoires dans le contexte burkinabè. Elle s'appuie sur des données secondaires, 3880 femmes âgées de 15 ans et plus, issues de l'enquête sur l'Interaction entre Politiques Publiques, Migrations et Développement (IPPMD) au Burkina Faso en 2014 collectées auprès de 2200 ménages. L'analyse a été à la fois descriptive (bivariée et multivariée) et explicative (régression logistique). Les résultats montrent que le statut social de la femme est négativement associé à l'émigration internationale des femmes. Les émigrées sont celles qui ont un statut faible. Elles résident en milieu rural, dans des ménages ayant une expérience migratoire et un niveau de vie faible. Le statut social de la femme explique le départ vers l'étranger chez les femmes Burkinabè et contribue à l'exacerbation des flux migratoires au Burkina Faso. Le sexe du chef de ménage et la taille du ménage constituent un autre groupe de variables qui influencent directement l'émigration des femmes mais aussi 
de façon indirecte via le statut de celles-ci. En conclusion, cette étude aurait contribué à une compréhension plus large sur les migrations féminines au Burkina Faso. Au regard de ces résultats, l'amélioration des conditions de vie des femmes et l'analyse de l'impact de l'émigration internationale de cellesci sur certains secteurs de développement local telles que la santé et l'éducation constituent des pistes à explorer afin de contribuer à l'autonomisation des femmes d'une part et de tirer les avantages qu'offre la migration d'autre part.

Mots-clés: Femme, Statut social, émigration internationale, Migration, Burkina Faso

\title{
Statut social et émigration internationale des femmes au Burkina Faso
}

\author{
Ousmane Barry \\ Université Joseph Ki-Zerbo, Burkina Faso. Institut National de la Statistique \\ et de la Démographie (INSD), Burkina Faso
}

\section{Honoré Mimche}

Patrice Tanang Tchouala

Hamidou Kone

Institut de FOrmation et de Recherche Démographiques (IFORD), Yaoundé, Cameroun

\begin{abstract}
The social status of women is a criterion of differentiation in the field of international female migration. However, the literature is not very prolific on the relationship between this and the practice of migration. This study aims to document the links between the status of women and their migratory behavior in the Burkina Faso context. The study is based on secondary data, 3,880 women aged 15 and over, from the survey on the Interaction between Public Policies, Migration and Development (IPPMD) in Burkina Faso in 2014 collected from 2,200 households. The analysis was both descriptive (bivariate and multivariate) and explanatory. Results show that the social status of women is negatively associated with the international emigration of women. The emigrants are those who have a weak status. They live in rural areas, in households with migratory experience and a low standard of living. The social status of women explains the departure abroad among Burkinabè women and contributes to the exacerbation of migratory flows in Burkina Faso. The sex of the head of the household and the size of the household constitute another group of variables that directly influence the emigration of
\end{abstract}


women but also indirectly via their status. In conclusion, this study would have contributed to a broader understanding of female migration in Burkina Faso. Based on these results, improving the living conditions of women and analyzing the impact of their international emigration on sectors of local development such as health and education are avenues for consideration to explore in order to contribute to the empowerment of women on the one hand and to reap the benefits of migration on the other.

Keywords: Woman, Social status, international emigration, Migration, Burkina Faso

\section{Introduction}

La migration est un phénomène universel qui touche tous les continents et qui est au cœur de l'actualité tant dans les pays du Nord que dans ceux du Sud (Brachet, 2007; Vause, 2012). À l'échelle mondiale, le nombre des migrants internationaux ne fait qu'augmenter : ils étaient 281 millions en 2020 contre 173 millions en 2000. Les femmes représentaient environ la moitié de ceux-ci (Nations Unies, 2020). Divers facteurs socioéconomiques, politiques et culturels entrainent cette augmentation et une diversification inédite des flux migratoires, notamment en Afrique Subsaharienne (Brachet, 2007). Tant pour les migrations internationales qu'internes, le constat est relativement le même: le déséquilibre économique entre régions, les conflits internes, l'absence de développement économique et social, la pauvreté monétaire, l'insuffisante scolarisation, le manque de professionnalisation, la quête du mieux-être de soi-même et de sa famille, les regroupements familiaux sont autant de raisons qui expliquent encore l'exacerbation des migrations (Brachet, 2007).

Bien que faisant, de plus en plus, l'objet de nombreuses études scientifiques par des chercheurs, la migration internationale reste le phénomène démographique le plus mal mesuré et le moins connu, en particulier en Afrique (Tabutin \& Schoumaker, 2020). Même si, au cours des dernières années, les recherches sur les migrations ont intégré davantage la dimension du genre (Toma, 2014), il demeure que les femmes ont longtemps été considérées comme totalement «passives » ou des «suiveuses » dans le processus migratoire. Dans la littérature, l'on distingue plusieurs travaux sur les migrations féminines qu'on pourrait regrouper en quatre types. D'abord ceux qui portent sur les relations de genre et la migration (Ambrosetti et al., 2008; Ba, 1998; Catarino \& Morokvasic, 2005; Chort et al., 2020; Lutz, 2010; Morokvasič, 1986; Pessar, 1999; Sakho \& Dial, 2010; Vause, 2009, 2012) et ensuite, les travaux renseignant sur les déterminants de la migration (Findley, 1989; Kenne, 2011; Gael Le Jeune et al., 2013; Lesclingand \& Hertrich, 2017; Néya, 2016). Aussi, d'autres mettent en avant le phénomène de féminisation 
des migrations (Mimche et al., 2005; Miranda et al., 2011; Vause et al., 2015) et enfin, les recherches qui font ressortir l'effet de la migration sur le statut de la femme (Moujoud, 2008).

Cependant, les travaux réalisés dans le contexte d'Afrique Subsaharienne se caractérisent par leur caractère plutôt général faisant que de nombreuses dimensions et approches méthodologiques d'analyse de l'émigration internationale féminine demeurent mal, insuffisamment ou non explorée. Aussi, les études faisant référence au statut de la femme sont relativement peu nombreuses (Assogba, 1992; Findley, 1989).

Au Burkina Faso, certes, la migration a fait, relativement, l'objet de nombreuses études scientifiques (Beauchemin, 2011; De Lange, 2007; Henry et al., 2003; Gaël Le Jeune, 2007; OIM, 2016; Ouedraogo, 2007; Zourkaleini, 2007; Zourkaléini \& Piché, 2005) mais peu d'entre elles ont abordé la problématique de l'émigration féminine. De même, les recherches l'ayant abordée, notamment celles de Le Jeune (2007), Le Jeune et al. (2013) et de Néya (2016), l'ont fait dans une approche transversale, sous l'angle des relations de genre et des effets de celle-ci sur les femmes sans évaluer l'effet net du statut de la femme sur la propension à migrer. Le statut de la femme influence-t-il l'émigration féminine au Burkina Faso ? Si oui, comment ? Qui sont celles qui émigrent ? Comment varie cette émigration internationale selon le statut des femmes? A travers quels mécanismes le statut des femmes influence-t-il leurs comportements migratoires?

La situation des femmes, en particulier celles du Burkina Faso, a connu des changements aux niveaux social, économique, politique et culturel (INSD, 2009; Rouamba, 2011). Le niveau d'instruction de la population féminine s'est amélioré au cours des dernières décennies. La participation à l'activité économique et politique (Omeyaka, 2018), l'âge au premier mariage des femmes et la proportion de celles qui demeurent définitivement célibataires ne font qu'augmenter. Ainsi, lorsqu'elles sont économiquement (Félix et al., 2017) et socialement autonomes, les femmes deviennent de puissants vecteurs de changement. La procréation, l'éducation des enfants et la « gardienne de la maison » qui formaient l'identité et la fonction unique de la femme tendraient à relever du passé. Le statut social de la femme résulterait de tous ces différents changements, il figure parmi les facteurs explicatifs de la dynamique démographique qui ont particulièrement retenu l'attention des chercheurs en Afrique (Assogba, 1989, 1990, 1992; Kamgno, 1993; Rakotondrabe, 2004; J.R. M. Rwenge, 2003, 2007; M. Rwenge, 1995).

Compte tenu de ce qui précède, cette recherche vise à documenter les liens entre le statut des femmes et leurs comportements migratoires dans le contexte burkinabè. 
Elle s'articule successivement autour de la population étudiée, d'une synthèse de la littérature existante sur la migration des femmes, des aspects méthodologiques, des principaux résultats et de la conclusion.

\section{Aspects Contextuels du Pays}

Le Burkina Faso est un pays sahélien, enclavé, situé au cœur de l'Afrique occidentale dans la boucle du Niger. Il est membre de la Communauté Economique des Etats d'Afrique de l'Ouest (CEDEAO), un espace qui se caractérise par la libre circulation des personnes, des biens et des services entre les Etats membres. Le pays a signé également des conventions internationales relatives à la libre circulation (Ministère de l'économie, des finances et du développement, 2017). En outre, il dispose d'une Stratégie Nationale de Migration (SNMig), adoptée en février 2017, qui est la politique de référence en matière de migration.

La dynamique démographique est caractérisée par une fécondité et une mortalité assez élevées et un solde migratoire négatif (INSD, 2009, 2011). Les femmes ont un pouvoir économique très faible du fait de leur faible accès et contrôle aux facteurs de production et aux ressources (Ministère de la promotion de la femme, 2009; Sigrun, 2004). L'émigration des femmes seraient conditionnées par plusieurs facteurs tels que la situation matrimoniale, le niveau d'instruction, la situation d'activité, le revenu, le statut par rapport à la maternité entre autres. En effet, le système familial et social est fondé sur le patriarcat et donc les décisions importantes, telle que l'émigration d'une femme par exemple, au sein du ménage ou dans la sphère familiale revient au chef. Ce pouvoir de la prise de décision au sein du ménage pourrait avoir une double implication: d'une part, il a une influence sur la position sociale des femmes qui tendrait à être toujours inférieure à celle des hommes; et d'autre part, il constitue un blocage pour leur liberté notamment celle relative à la libre circulation.

Avec une pluralité ethnique qui donne lieu à une diversité de pratiques socioculturelles et des systèmes de rapport de genre variés, on retrouve des ethnies où la femme : (1) a un pouvoir économique et un pouvoir de décision, (2) a un certain pouvoir de décision mais son pouvoir économique est faible, (3) n'a pas un pouvoir de décision important mais peut disposer des ressources propres (Kobiané, 2007). Ainsi, au regard des spécificités des groupes ethniques du pays, on peut déduire que le fait pour une femme d'appartenir à l'un ou l'autre groupe peut manifestement avoir un impact sur son statut au sein dudit groupe et partant de la société dans laquelle elle vit. Le fait d'avoir un pouvoir économique et/ou décisionnel constitue, sans doute, un atout considérable dans un projet migratoire. 


\section{Revue De La Litterature}

L'émigration, féminine, est un phénomène dont l'occurrence dépend d'un ensemble d'éléments très complexes, à la fois individuels et collectifs, souvent interactifs. Plusieurs approches théoriques et empiriques ont été mobilisées par de nombreux chercheurs, de disciplines variées, pour comprendre ledit phénomène.

\subsection{Quelques approches explicatives}

L'approche genre, un objet d'analyse important pour une meilleure compréhension des changements sociaux autant dans la société de départ, de transit que celle d'immigration. Pendant longtemps, les rapports de genre furent ignorés et le neutre au masculin fut considéré comme suffisamment légitime pour représenter tous les migrants. Pour (RoulleauBerger, 2010), la question du genre dans les migrations est longtemps restée un impensé. Le texte de Morokvasic paru en 1984 dans le numéro spécial de l'International Migration Review sur les migrations féminines fut le point de départ obligé en ce qui concerne la théorisation du rôle des femmes dans la migration (Piché, 2013). C'est ainsi que le rôle des rapports de genre dans les décisions migratoires a été abordé dans l'optique d'une théorie spécifique de la place des femmes dans la société (Nganawara, 2017).

Toutefois, Ba (1998) avait déjà précisé qu'il ne s'agit pas d'envisager strictement l'étude des femmes en tant que groupe spécifique mais de manière transversale aborder également la question de l'homme. Pour certains auteurs, la migration des femmes s'inscrit donc dans des rapports de pouvoir et les recherches examinent non seulement la manière dont ces rapports façonnent les décisions migratoires des femmes, mais aussi comment la migration féminine peut changer le système patriarcal (Gael Le Jeune et al., 2013; Piché, 2013). Bien que les migrations féminines rythment aux pas des rapports de genre dans de nombreux pays du monde en général et ceux africains en particulier, il existe néanmoins des déplacements légitimés et autorisés : les regroupements familiaux.

L'approche sociologique, elle regroupe en son sein plusieurs théories dont celles des migrations féminines dans le cadre du regroupement familial, du pull/push factors et des réseaux migratoires entre autres.

Le regroupement familial fournit un cadre à l'étude des migrations féminines. Le postulat est que les jeunes hommes célibataires ou mariés émigrent d'abord seuls pour des raisons de travail et font venir ensuite leurs fiancées ou épouses. Les femmes qui migrent dans ce cadre sont présentées comme des «suiveuses", dont le départ est fondé exclusivement sur la décision du conjoint émigré. Leur image est celle de personnes à charge plutôt que celle d'actrices de la migration (Tall \& Tandian, 2010). Cependant, les migrations féminines africaines présentent plusieurs caractéristiques. Bisilliat 
(1983) en note déjà trois types : ou bien ce sont des filles qui partent vers l'âge de 15 ans pour subvenir aux besoins de leurs familles ou bien ce sont des femmes chefs de famille qui quittent leur village faute de terres ou d'emplois rémunérateurs, ou bien, enfin, ce sont des femmes mariées qui suivent leurs maris lorsque ces derniers émigrent (Bisilliat, 1983).

A côté de ces formes de migration, Mankou (2011) note l'apparition d'un nouveau phénomène social de migration féminine en Afrique : la cybermigration maritale. Il s'agit, pour l'auteur, d'une modalité de mobilité contemporaine qui concerne principalement des jeunes filles en quête d'un meilleur statut social. Ces dernières s'auto exclues du marché matrimonial «normal » de leur pays, jettent leur dévolu sur le «Blanc» qui est perçu comme un occidental, figure du mari idéal. Ce qui confère un meilleur statut à la jeune fille et lui permet de venir en aide à ses proches restés au pays (Mankou, 2011).

Nonobstant, même si l'émigration des femmes demeure un phénomène méconnu (Mimche et al., 2013), certains travaux montrent qu'on assisterait à un développement sans précédent des migrations autonomes ${ }^{1}$ des femmes en Afrique (Adepoju, 2002; Antoine \& Sow, 2000; Bocquier \& Traoré, 2000; Mimche et al., 2013).

Quant à la théorie de pull/push factors, Lee (1966) considère que dans chaque milieu de l'espace migratoire, il y a des éléments répulsifs, attractifs et neutres (Lee, 1966). Alors, il part du principe que la migration est un calcul individuel fondé sur les facteurs d'attraction (lieu de destination) et les facteurs de répulsion (lieu d'origine) et postule que « la migration est causée à la fois par des facteurs positifs qui caractérisent les aires possibles de destination et par des facteurs négatifs aux lieux d'origine ou de résidence actuelle. Ainsi, tant la zone de départ que la zone d'arrivée sont caractérisées par un ensemble de facteurs positifs, ou facteurs d'attraction ou de rétention (pull factors) et de facteurs négatifs ou forces de répulsion (push factors) » (Caselli et al., 2003). Pour cet auteur, les contacts personnels et les sources d'informations disponibles sont entre autres des facteurs qui interviennent dans le processus de migration. Toutefois, quelques critiques ont été formulées sur la théorie parmi lesquelles la prédominance des facteurs micro-individuels.

L'approche systémique pourrait être d'une grande importance dans l'explication de l'émigration internationale féminine en ce sens que non seulement la migration est complexe en elle-même mais également les faits sociaux relatifs aux femmes le sont aussi. L'avantage de cette approche dans l'appréhension des faits migratoires est qu'elle est une démarche à la fois

${ }^{1}$ La migration "autonome" des femmes est définie dans ces travaux comme une migration visant à satisfaire les besoins économiques individuels des femmes. Elle résulterait principalement de la "féminisation de la pauvreté" qui affecterait particulièrement les milieux ruraux dans ces régions. 
analytique et synthétique, détaillante et englobante. Selon Cambien (2008), la naissance de l'approche systémique est en partie liée à la prise de conscience, notamment au sein du champ scientifique, de la complexité croissante du monde. En d'autres termes, plus le monde devient complexe et plus l'Homme tend à développer une conscience plus aiguë de cette complexité (Cambien, 2008).

Au-delà de la théorie, des études empiriques ont été menées sur les migrations internationales s'intéressant aussi bien à la mesure qu'à l'explication du phénomène.

\subsection{Facteurs explicatifs}

Les chercheurs ont accordé, et continuent d'accorder de plus en plus et dans divers domaines, de l'intérêt au statut de la femme. Cet intérêt est justifié, le plus souvent, par l'importance du rôle de la femme dans le processus de développement et/ou par les inégalités entre sexes ou encore par la théorie de la transition démographique d'un pays, surtout ceux d'Afrique. Vu sous cet angle, le statut de la femme a également suscité l'intérêt des chercheurs dans les études démographiques (Assogba, 1989, 1990, 1992; Kamdem, 2006; Rakotondrabe, 2004; J.-R. M. Rwenge, 2003, 2007; M. Rwenge, 1995). Cet intérêt des chercheurs pour la féminisation du discours sur les migrations est récent bien que la féminisation des migrations ne soit pas tout à fait un phénomène nouveau (Vause, 2012).

Avant toute tentative de prise en compte de cet indicateur dans une étude, il est important de noter les problèmes inhérents à la mesure et à la conceptualisation même du statut de la femme. Bien que reconnu par divers auteurs comme un important facteur de changement démographique (Assogba, 1989; Vallin, 1988), le statut de la femme, jusqu'à un passé récent, était souvent réduit au niveau d'instruction de la femme et/ou à son activité économique (Assogba, 1990; Kamdem, 2006; J.-R. M. Rwenge, 2007). Cependant, cette manière de simplifier une réalité très complexe a fait l'objet de nombreuses critiques (Assogba, 1992; Kamdem, 2006; Oppong, 1980; Rakotondrabe, 2004; J.-R. M. Rwenge, 2003, 2007).

Pour Assogba (1992), le statut social de la femme étant multidimensionnel et le représenter par une seule variable donnerait une vision trop partielle voire inexacte de la réalité. Rakotondrabe (2004) se résume en disant qu'il est difficile, voire impossible d'appréhender le statut social de la femme à partir d'une variable unique. Kamdem (2006), quant à elle, soulève une difficulté majeure que posait de mesurer le «statut social de la femme » par son activité professionnelle. Cette difficulté résiderait dans le problème de quantification des travaux domestiques qui, dans plusieurs études, ne sont pas prises en compte. Dans le même ordre d'idée, Rwenge (2007) pense qu'une 
femme de niveau d'instruction élevé peut remettre en question son statut social traditionnel sans pour autant avoir nécessairement un statut élevé.

Pour mieux opérationnaliser le statut social de la femme et intégrer les différences entre hommes et femmes dans les indicateurs de mesure du concept, les chercheurs ont ainsi été amenés à développer d'autres approches. Ce qui a permis de réaliser des progrès théoriques dans le cadre de la mesure du concept de statut social de la femme, mais ces progrès n'ont pas été suivis (sinon très peu) par des applications pratiques (J.-R. M. Rwenge, 2007).

Parmi ces études figure celle de Rakotondrabe (2004). Elle indique la nécessité de la prise en compte de ses multiples dimensions et des variations de ces dernières selon la sphère d'intervention de la femme. Elle distingue alors deux types de statut: le statut dans la société ou le statut public et le statut dans le ménage ou statut privé. Le statut public correspond à la position de la femme en tant qu'individu vivant dans la société et de ce fait reflète sa position relative par rapport aux autres individus de la même société. Pour opérationnaliser le statut public de la femme, elle a utilisé les variables : relatifs au niveau d'instruction et l'activité de la femme, sa religion, son âge, le niveau d'instruction et l'activité de son mari ainsi que le nombre d'enfants nés vivants. S'agissant du statut privé, il prend en compte la position relative de la femme par rapport à son mari ou un autre membre vivant dans le ménage. La différence d'instruction et d'activité des conjoints, l'écart d'âge entre époux et la position de la femme dans le ménage sont les variables mobilisées pour la mesure du statut privé.

Par ailleurs, le milieu et la région de résidence constituent des variables révélatrices des diverses disparités concernant l'évolution d'un phénomène sociodémographique. Plusieurs études empiriques ont mis en relief le milieu de résidence et l'émigration (Gael Le Jeune et al., 2013; Zourkaleini, 2007; Zourkaléini \& Piché, 2005). On assiste à une intensification de l'exode rural des femmes et parallèlement des migrations féminines. Nombreux sont les auteurs qui les qualifient d' "autonomes » à la lumière d'un certain nombre d'indicateurs (Adepoju, 2002; Gael Le Jeune et al., 2013). Au Burkina Faso par exemple, Zourkaléini (2007) montre qu'en ce qui concerne l'émigration internationale, plus de $90 \%$ des hommes et des femmes partent des régions pauvres. Dans le même sens, Le Jeune et al. (2013) montrent que le milieu de résidence du conjoint est déterminant pour l'émigration des femmes en union. Sarr et al. (2009) expliquent aussi que de nombreuses migrations féminines se font des zones rurales vers les villes ou le péri-urbain et ce pour plus d'une raison notamment la scolarisation, la professionnalisation, l'écoulement des produits agricoles.

En plus, la revue de la littérature a permis d'identifier des variables explicatives pertinentes en lien avec le comportement migratoire des femmes. 


\section{Il s'agit de la religion, l'ethnie, l'expérience migratoire du ménage, le sexe du chef de ménage, l'âge et le statut matrimonial de la femme.}

En effet, selon Zidnaba (2016), dans sa thèse sur la migration internationale et le développement au Burkina Faso, deux facteurs justifient le différentiel selon l'ethnie: la proximité géographique et les habitudes ethniques. Pour Dabiré (2004), l'intensité de la migration peut varier d'une ethnie à une autre et n'est pas spécifique à une ethnie. Cependant, des enquêtes antérieures et anciennes (1960-61, 1991) réalisées au Burkina Faso montrent que la religion et l'ethnie sont associées à la migration. Les Mossis, Gourounsis et Bissas semblent avoir une propension à migrer plus forte que les autres ethnies du pays tant au niveau interne qu'international. Aussi, en cherchant les déterminants familiaux de l'émigration dans deux régions du Burkina Faso, Dabiré (2004) est arrivé au résultat selon lequel l'expérience migratoire du ménage exerce une influence sur les départs des membres du ménage. Il ajoute qu'en général les migrations burkinabè que ce soit en direction des zones rurales, urbaines ou à l'étranger suivent des filières établies par les primo migrants, ceux qui ont déjà migré transmettant les informations nécessaires aux autres. Dans le cas spécifique des migrations burkinabè, Hazard B. (2007), cité par Zidnaba, (2016) montre qu'en Italie, elles se sont construites dans l'optique des réseaux (Zidnaba, 2016). Le rapport ${ }^{2} \mathrm{~d}^{\text {'analyse }}$ du projet Interactions entre les Politiques Publiques, les Migrations et le Développement (IPPMD) réalisé au Burkina Faso en 2014, quant à lui, montre que les projets d'émigration sont plus fréquents dans les ménages qui ont une expérience migratoire, surtout dans ceux recevant des transferts de fonds et dans ceux avec des migrants de retour.

En Afrique, peu sont les ménages où les femmes sont chefs et même si elles le sont, cela est dû à des circonstances particulières telles que le célibat, le divorce, le veuvage ou encore l'émigration des hommes qui les ont contraintes à devenir chefs de ménages (Oso \& Catarino, 1997). D'autres travaux de recherche, plus anciennes, dont ceux de Gregory et al. (1986) ont conclu à la formulation d'hypothèses sur l'existence de stratégies collectives de migration. Pour ces derniers, les charges et responsabilités familiales ou sociales sont avancées par presque tout le monde pour expliquer le fait que certains ne soient pas partis ou ne partent pas. En effet, si la migration est souhaitée par le ménage, elle ne doit pas être un élément déstructurant de la société. Une exigence des ménages en zone rurale africaine est la nécessité de leur maintien comme unité de production et de reproduction dans le village d'origine pour gérer le patrimoine ancestral (terre, fétiche, etc.), assurer la retraite des personnes âgées et perpétuer la tradition. Dans cette optique, la

2 Ce rapport, publié le 26 mai 2017, est disponible sur https://www.oecdilibrary.org/docserver/9789264275003fr.pdf?expires=1526122566\&id=id\&accname=guest \&checksum=C8C0B61BEF4C082090D1D4F3DFC477E1 
migration doit se faire dans le respect du maintien de la famille ou du ménage en milieu rural dans les fonctions essentielles ci-dessus évoquées (Dabire, 2004). Concernant l'âge, la distribution des femmes migrantes révèle en premier lieu l'importance de la migration dans le groupe d'âge 15-24 ans, puis de façon moins prononcée chez les femmes de plus de 50 ans (Bréant, 2012). L'étude de Marsicano et al. (2011) sur l'entrée dans la sexualité des migrants d'Afrique Subsaharienne en France montre par exemple que les femmes arrivent plus jeunes que les hommes (âge médian à la migration de 19,9 ans vs 22,7 ans). Au Togo, une étude nationale menée par Kombieni (2015) montre qu'en moyenne $27 \%$ de la population des femmes émigrantes ont entre 20 à 30 ans (Kombieni, 2015).

\section{Aspects Methodologiques}

Dans cette partie sont présentés les hypothèses de travail, les aspects conceptuels, les données utilisées ainsi que les variables et les méthodes d'analyse.

\subsection{Hypothèses}

Compte tenu des résultats de la synthèse de la littérature, les hypothèses principales, dans le cadre de cette étude, sont les suivantes :

H1 : Au Burkina Faso, l'émigration solitaire des femmes demeure un phénomène marginalisé. Une amélioration du statut social de la femme entraine en principe une augmentation de son pouvoir économique et partant de son pouvoir décisionnel. De même, l'amélioration du statut va de pair avec l'activité professionnelle, on peut supposer que les femmes de statut élevé appartiennent à des catégories socioprofessionnelles spécifiques et sont de ce fait prédisposées pour des raisons socioprofessionnelles être portées à se mouvoir et même à l'international. Ainsi, la première hypothèse est que, les femmes ayant un statut élevé sont plus susceptibles d'émigrer ou d'avoir l'intention d'émigrer à l'international que celles ayant un statut faible.

H2 : La région et le milieu de résidence dans lesquels résident les femmes sont des facteurs qui impactent leurs sphères de décisions et les informations médiatiques (exposition aux médias et aux réseaux sociaux) ainsi que leur statut au sein de la société. De ce fait, la deuxième hypothèse postule que la région et le milieu de résidence ont, chacune, en plus de l'effet " net » direct, un effet indirect sur le statut d'émigration international via le statut de la femme.

H3 : Les ménages qui disposent déjà de migrants internationaux confèrent à leurs membres une grande expérience de la migration constituée d'informations sur les risques et avantages de l'émigration mais aussi d'opportunités d'accueil, d'insertion et de la géographie des lieux avantageux de migration. Aussi, dans les ménages de niveau de vie faible, le chef de 
ménage et le ménage en général peut être amené à solliciter la contribution de leurs membres. Ce qui peut pousser aux émigrations. On peut également penser que lorsque c'est une femme qui est le chef de ménage, la logique patriarcale est peu prédominante et il se développe une solidarité féminine en ce qui concerne les faits sociaux. Par conséquent, la troisième hypothèse soutient que l'expérience migratoire et le niveau de vie du ménage ainsi le sexe du chef de ménage et la taille du ménage ont non seulement un effet "net» direct sur le statut d'émigration international mais aussi un effet indirect via le statut de la femme.

\subsection{Aspects conceptuels}

Les aspects conceptuels portent sur la définition des concepts de statut social de la femme, de l'émigration internationale ainsi que sur le schéma conceptuel de l'étude.

\subsubsection{Définition des concepts clés}

Le statut social de la femme est un concept complexe, dynamique, multidimensionnel et multidisciplinaire (Assogba, 1989, 1990, 1992; Rakotondrabe, 2004; J.-R. M. Rwenge, 2007). Il n'est donc pas aisé de donner une définition claire et précise de ce concept. C'est pourquoi, pour Assogba (1990) et Rakotondrabe (2004), la définition de la variable «statut de la femme » de plus en plus fréquente dans plusieurs études, constituerait à elle seule un sujet de recherche. On rencontre ainsi dans la littérature une diversité de définitions variant selon les disciplines, les auteurs, les objectifs et les contextes. Dans le dictionnaire Larousse 2010, le statut social est défini comme «Situation de fait, position par rapport à la société ». Ce statut est avant tout social (Kamgno, 1993). Certains auteurs ont remis cette définition en cause, notamment Assogba (1989) et Rakotondrabe (2004). Pour le premier, cette définition peut certainement s'adapter au cas du statut de la femme mais est insuffisante par le fait qu'elle manque de précision. Pour le deuxième, la notion de position ou de situation est trop large, peu opérationnelle et ne suffit pas pour caractériser un individu en ce sens qu'elle porte à croire que le statut de la femme se rapporte au fait d'être femme. Pourtant, ce statut, cette position sociale renvoie aux rôles et aux attentes ainsi qu'aux droits et aux devoirs de la femme dans la société. Il en donne la définition suivante : «une position sociale qui lui procure un certain prestige au niveau de la société, et qui conditionne son accès aux ressources, sa faculté de contrôle de ces ressources et l'exercice d'un pouvoir ou d'une possibilité de participer à la prise de décision (au sein du ménage) 》(Rakotondrabe, 2004 : p.37). Ainsi pour l'auteur, les différents éléments intégrés dans la définition du statut sont des sources d'inégalités aussi bien entre les femmes 
elles-mêmes qu'entre les femmes et les hommes et vont caractériser chacune des femmes.

Dans le cadre de cette étude, le statut social de la femme doit être compris comme sa position au sein de la société qui pourrait déterminer sa capacité à se mouvoir. Pour opérationnaliser le concept du statut social de la femme, un indicateur a été construit à partir de plusieurs variables. L'indicateur est donc le statut de la femme.

L'émigration internationale féminine est considérée dans cette étude comme le départ, pour un pays étranger ou l'intention de partir du Burkina Faso, d'un membre du ménage de sexe féminin, pour une période d'au moins trois mois, quel qu'en soit le motif. L'émigrante ou l'émigrée est alors toute personne de sexe féminin qui a effectué une émigration, c'est-à-dire une personne de sexe féminin qui a quitté le ménage pour un autre pays ou qui a l'intention de partir vivre dans un autre pays. La définition donnée à ce concept dans ce travail diffère donc de celle du rapport d'analyse de l'enquête IPPMD qui n'a pas pris en compte l'intention migratoire.

\subsubsection{Modèle conceptuel}

Le statut social de la femme, sous l'effet des contextes résidentiel, socioculturel et des caractéristiques du ménage, influence directement l'émigration internationale des femmes et indirectement via la prise de décision. Notre modèle conceptuel se présente alors comme suit : 


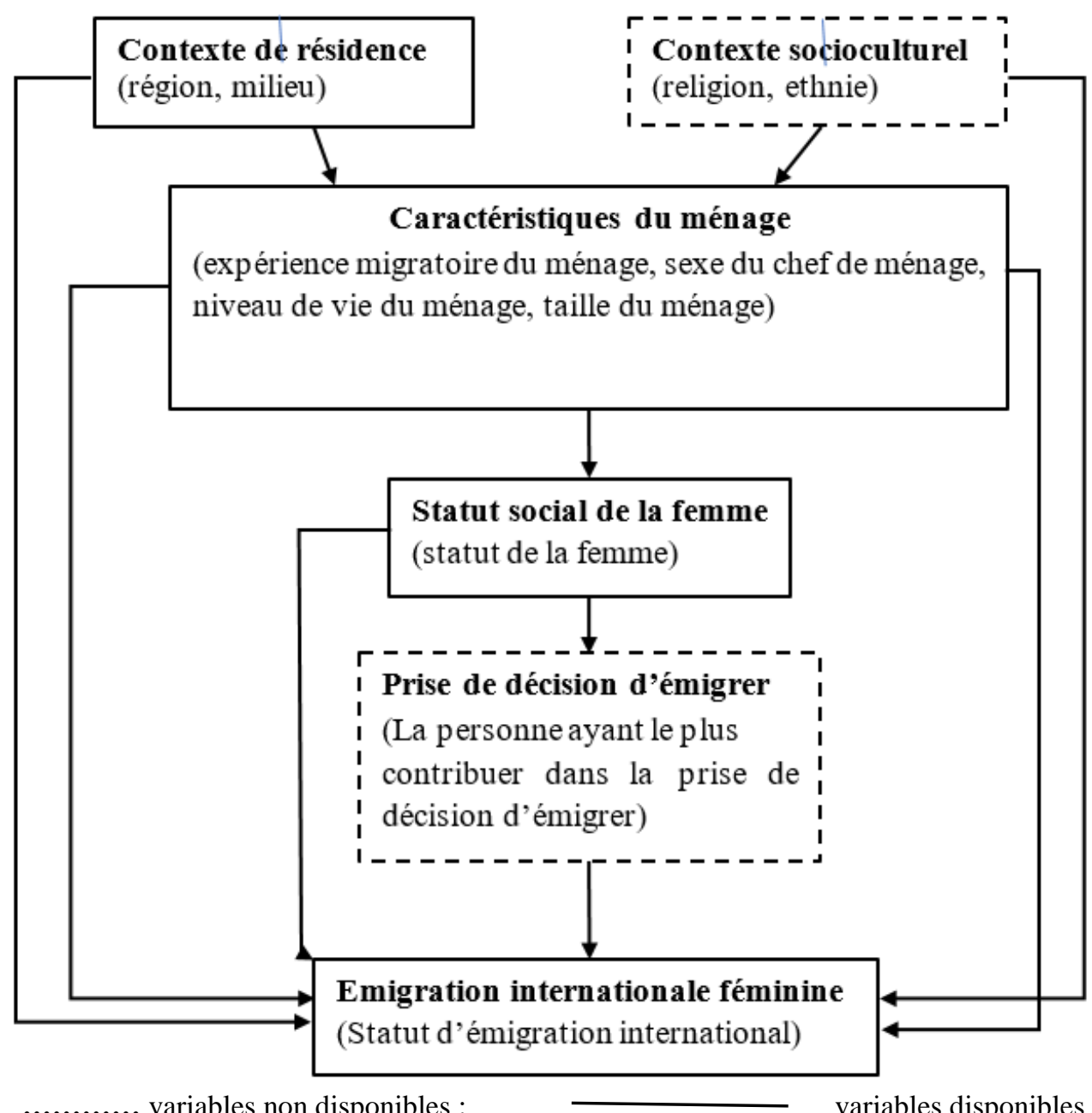

\subsection{Données et variables d'étude}

\subsubsection{Source de données}

Les données utilisées sont issues de l'enquête sur l'Interactions entre Politiques Publiques, Migrations et Développement (IPPMD) qui a permis de recueillir des observations entre avril et mai 2014 au Burkina Faso. Elle a couvert tout le territoire national. Les enquêtes auprès des ménages et des communautés du Burkina Faso sur l'IPPMD se sont appuyées sur un plan d'échantillonnage composé de deux groupes stratifiés à deux niveaux et représentatifs à l'échelle nationale. Les 405 communes et districts urbains que compte le pays (désormais désignés sous le terme de "communes » uniquement) ont en effet d'abord été répartis en deux strates. Le critère de la première strate était le caractère rural ou urbain de la commune, et celui de la seconde strate le niveau - faible ou élevé - de son taux de migration, d'après 
les données du Recensement général de la population et de l'habitation (RGPH) réalisé en 2006.

La population cible est constituée des femmes âgées de 15 ans et plus, qu'elles aient émigrées ou pas. Ainsi, la taille de l'échantillon est de 3880 femmes dont $64,1 \%$ sont mariées ou en union libre, $27,84 \%$ des célibataires et $8,07 \%$ des divorcées, veuves ou séparées. La proportion des femmes ayant moins 25 ans est de $34,12 \%$ et celle des femmes ayant plus de 35 ans est de $38,02 \%$. Il ressort aussi que plus de $63 \%$ des femmes de l'échantillon n'ont aucun niveau d'instruction et seulement moins de $6 \%$ ont une activité rémunérée. Ce qui caractérise l'échantillon est que moins de $6 \%$ des femmes sont des chefs de ménage.

\subsubsection{Variables}

Elles se répartissent en deux groupes à savoir : la variable dépendante et les variables indépendantes. Certaines variables ont subi un traitement et/ou des recodages, tandis que d'autres ont été créées à partir de la combinaison de plusieurs autres variables de la base de données.

Le focus de cette étude est d'analyser le statut migratoire de la femme - variable dichotomique - incluant l'expérience d'émigration internationale et/ou l'intention d'émigrer de la femme.

Les variables indépendantes sont celles qui permettent d'élucider le phénomène étudié. Elles sont au total sept (07) variables. Le statut de la femme, variable indépendante principale, est construit à partir du niveau d'instruction, l'activité, le statut matrimonial, l'âge et le lien de parenté de la femme avec le chef de ménage. Aussi, le choix de ces variables se justifie par l'existence d'une forte association entre celles-ci dans le contexte de notre étude. La méthode utilisée pour sa construction est la méthode de l'Analyse à Composantes Principales (ACP) avec la commande «factor». Ainsi, la variable statut de la femme a deux modalités compte tenu du faible effectif des femmes émigrées : statut faible et statut élevé. La modalité statut faible prend en compte essentiellement les femmes n'ayant aucun niveau d'instruction, mariées ou en union libre, exerçant des activités non rémunérées, âgées de moins de 35 ans et qui sont épouses ou filles du chef de ménage. En effet, plus de $86 \%$ de ces femmes n'ont aucun niveau d'instruction, $88,82 \%$ sont épouses ou filles du chef de ménage et $63,48 \%$ exercent des activités non rémunérées. Quant à leur statut matrimonial, $79,42 \%$ sont mariées ou en union libre. Plus de $74 \%$ de ces femmes ont également moins de 35 ans. Les femmes de statut élevé se caractérisent essentiellement par leur niveau d'instruction, leur statut d'activité et leur âge. Plus de $62 \%$ de ces femmes ont au moins un niveau d'instruction secondaire. Plus de la moitié de ces femmes ont plus de 35 ans et $42 \%$ exercent une activité rémunérée. 
Les autres variables indépendantes sont la région et le milieu de résidence, le sexe du chef de ménage, l'expérience migratoire du ménage, le niveau de vie et la taille du ménage.

\subsection{Méthodes d'analyse}

S'agissant de la vérification des hypothèses, deux méthodes d'analyse ont été utilisées : l'analyse descriptive et l'analyse explicative.

Au niveau descriptif, l'analyse bivariée a permis de vérifier la liaison ou l'association entre la variable dépendante et chacune des variables indépendantes contrôlée par le statut de femme. L'évaluation de la liaison entre ces variables est faite à partir des tableaux de contingence, suivi d'un test de Chi2. Le seuil retenu pour la description de l'association des variables est de 5\%. Aussi, l'Analyse Factorielle des Correspondances Multiples (AFCM) a permis de positionner dans un repère orthonormé les différentes modalités de chaque variable et de caractériser les profils des femmes selon leur statut migratoire.

Au niveau explicatif, une régression logistique binomiale a permis de quantifier l'effet des variables indépendantes sur la variable dépendante, tout en tenant compte des autres variables introduites dans le modèle. L'effet du statut de la femme sur l'émigration internationale féminine, est établi, dans ce cadre en présence d'autres facteurs ou variables indépendantes. Le modèle de régression logistique fournit à cet effet plusieurs statistiques, entre autres :

- Le khi-deux $(\chi 2)$ du modèle et la probabilité (significativité) P qui lui est associée, qui permettent d'évaluer la qualité du modèle. Le khideux permet également d'hiérarchiser les facteurs explicatifs. Cette hiérarchisation se fait à l'aide des contributions de ces variables à l'explication du phénomène étudié. La contribution Ci d'une variable i donnée se calcule à l'aide de la formule :

$\mathbf{C i}=(\chi \mathbf{2} \mathbf{f}-\chi \mathbf{2 i}) / \chi \mathbf{2 f}$ où $\chi \mathbf{2} \mathbf{f}$, et $\chi \mathbf{2 i}$ désignent respectivement la valeur du khideux du modèle final ou saturé et celle du khi-deux du modèle sans la variable i.

- les odds ratios (risques relatifs) et les probabilités (significativités) $\mathrm{p}$ qui leurs sont associées. Elles permettent d'identifier les variables qui influencent significativement le phénomène étudié et de mettre en exergue les inégalités face à l'émigration selon chacune des variables de l'étude. Le pouvoir explicatif d'une modalité d'une variable est jugé significatif si la probabilité p correspondante est inférieure à 5\% (seuil retenu). Une variable est dite déterminante dans l'explication du phénomène si au moins une de ses modalités a un pouvoir explicatif significatif. En guise de rappel, $\mathrm{p}=\mathrm{P}(\mathrm{Z}=1)$ étant la probabilité que l'évènement étudié (émigration) se réalise, 1-p est la probabilité que 
cet évènement ne se réalise pas et le modèle de régression logistique permet de poser l'équation :

$$
\begin{gathered}
\mathrm{Z}=\operatorname{Logit}(\mathrm{P})=\log \left(\frac{p}{1-p}\right) \square e^{Z}=\frac{p}{1-p} \square \mathrm{p}=\frac{e^{Z}}{1-e^{Z}} \\
\text { avec } \boldsymbol{Z}=\boldsymbol{b} \mathbf{0}+\boldsymbol{b}_{1} \mathrm{X}_{1}+\boldsymbol{b}_{2} \mathrm{X}_{2}+\cdots+\mathrm{b}_{\mathrm{k}} \mathrm{X}_{\mathrm{K}}+\boldsymbol{E} \text { où les } \mathrm{X}_{\mathrm{i}}(\mathrm{i}=1,2,3 \ldots \mathrm{k})
\end{gathered}
$$
sont les variables explicatives ; le b0 est le terme indépendant de l'équation qui exprime le niveau moyen pour toutes les valeurs des variables indépendantes $\left(\mathrm{X}_{\mathrm{i}}\right)$; les bi sont les coefficients de régression à estimer à partir des données mesurant l'effet net de la variable $\mathrm{X}_{\mathrm{i}}$ (ou de la modalité d'une variable) ; ils sont rattachés à chacune des variables indépendantes $\left(\mathrm{X}_{\mathrm{i}}\right)$ et $\mathcal{E}$ représente la variation aléatoire due à l'action des variables implicites agissant sur les variables indépendantes.

La statistique $\mathrm{e}^{\mathrm{z}}=(\mathrm{p} / 1-\mathrm{p})$ est le « risque relatif » ou Odds Ratio. Si la valeur de $\mathrm{e}^{\mathrm{z}}$ correspondant à une modalité $k$ donnée est inférieure à 1 , on dira que les femmes de la catégorie $k$ ont 1-e $\mathbf{e}^{\mathbf{z}}$ fois moins de chances que leurs homologues du groupe de référence d'émigrer. Un odds ratio supérieur à 1 signifie que ce risque est de $\mathbf{e}^{\mathbf{z}}$ fois plus que les femmes du groupe de référence.

\section{Resultats}

\subsection{Variation de l'émigration des femmes selon le statut de la femme}

L'examen de la répartition des proportions des femmes émigrées ou ayant l'intention selon le statut de la femme révèle une relation négative entre le statut de femme et le statut migratoire. En effet, parmi les femmes de statut faible, 5,34\% ont émigré ou ont l'intention d'émigrer contre 3,93\% chez celles de statut élevé (cf. annexe1). Les femmes de statut faible émigrent plus que celles ayant un statut élevé. Cette différence est statistiquement significative au seuil de $5 \%$.

\subsection{Contrôle de la relation entre le statut migratoire et le statut de la femme par les variables contextuelles et du ménage}

L'association entre le statut de la femme et le statut migratoire est significative au seuil de 5\% dans les régions Nord Soudanienne et Sud Soudanienne. On observe que la proportion des émigrées et les émigrantes de statut faible est plus grande dans la région Sud Soudanienne et Nord Soudanienne comparativement à la région Soudano-Sahélienne. Dans la région Nord Soudanienne, 5,67\% des femmes émigrées ou ayant l'intention d'émigrer ont un statut faible contre $2,80 \%$ qui ont un statut élevé. Concernant la région Sud Soudanienne, la proportion des femmes émigrées de statut faible ou ayant l'intention d'émigrer est de $6,78 \%$ contre $4,36 \%$ pour celles de statut élevé. Concernant le milieu de résidence, le contrôle de la relation entre le statut de la femme et le statut d'émigration des femmes par celui-ci laisse apparaître un écart entre les proportions des femmes émigrées de statut faible 
et celles de statut élevé, en milieu rural. Cet écart est moins prononcé en milieu urbain. Au seuil de 1\%, le statut de la femme est significativement associé au statut d'émigration en milieu rural. Ceci n'est pas le cas en milieu urbain.

S'agissant des variables méso, la relation entre le statut de la femme et le statut migratoire est significative au seuil de 5\% dans les ménages dont le chef est une femme. Elle l'est aussi dans le groupe des femmes dont les ménages d'appartenance ont un faible niveau de vie ainsi que dans le groupe des femmes résidant dans des ménages dont la taille est supérieure à six (06) membres. Cependant, bien que la relation ne soit pas statistiquement significative, on observe que, quel que soit le statut de la femme, l'émigration internationale féminine est presque exclusivement une pratique des femmes issues des ménages ayant une expérience migratoire, c'est-à-dire des ménages ayant enregistré soit un ou plusieurs émigrés, immigrés ou migrant de retour ou encore les trois à la fois.

\subsection{Profil des émigrées et émigrantes}

Le graphique 1 présente deux groupes de femmes à partir de l'analyse factorielle des correspondances multiples. Le premier groupe est positionné du côté positif du deuxième axe (axe vertical) et le deuxième du côté négatif du même axe. Dans le premier groupe, on y trouve les femmes résidant dans la partie Nord-Soudanienne, en milieu rural et dans des ménages de faible niveau de vie. Ces femmes ont un statut faible et leurs ménages ont une expérience migratoire. Elles sont celles qui forment le groupe des émigrées et émigrantes. S'agissant du second groupe, il est constitué des femmes résidant en milieu urbain et dans des ménages de niveau de vie élevé. Ce deuxième groupe contient également les femmes dont les ménages d'appartenance n'ont aucune expérience migratoire. Ces ménages sont dirigés par des femmes et celles qui y habitent habituellement ont un statut élevé. 


\section{Graphique 1 : Catégorisation des femmes selon leurs caractéristiques}

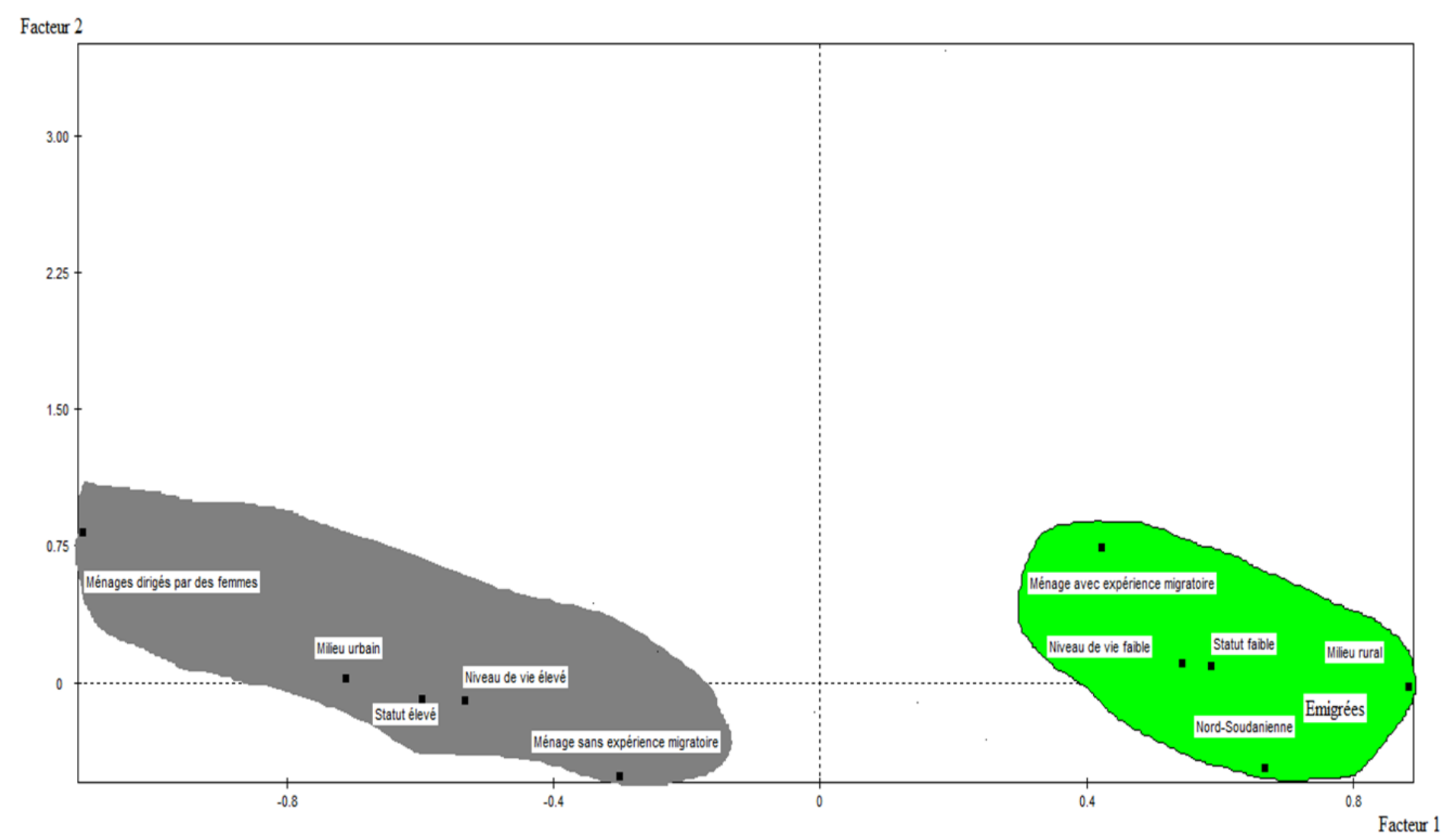




\subsection{Essai d'explication des différences de l'émigration internationale féminine}

\subsubsection{Evaluation du pouvoir discriminant du modèle : la courbe ROC}

La courbe ROC est une représentation graphique qui permet de mesurer le pouvoir discriminant des variables explicatives sur l'émigration internationale des femmes. Autrement dit, elle permet de s'assurer de la prise en compte de variables pertinentes pour l'explication du phénomène. Ainsi, les conditions se présentent comme suit :

\begin{tabular}{|l|l|l|l|}
\hline $\mathbf{A}(\mathbf{R O C})=\mathbf{0 , 5}$ & $\mathbf{A}(\mathbf{R O C})=[\mathbf{0 , 7} ; \mathbf{0 , 8}]$ & $\mathbf{A}(\mathbf{R O C})=[\mathbf{0 , 8} ; \mathbf{0 , 9}]$ & $\mathbf{A}(\mathbf{R O C}) \geq \mathbf{0 , 9}$ \\
\hline $\begin{array}{l}\text { Il n'y a pas de } \\
\text { discrimination }\end{array}$ & $\begin{array}{l}\text { La discrimination est } \\
\text { acceptée }\end{array}$ & $\begin{array}{l}\text { La discrimination est } \\
\text { excellente }\end{array}$ & $\begin{array}{l}\text { La discrimination est } \\
\text { exceptionnelle }\end{array}$ \\
\hline
\end{tabular}

En application de cette règle et au regard de la valeur de l'aire sous la courbe ROC (graphique ci-dessous), il ressort que la discrimination est excellente et par conséquent les variables explicatives sont pertinentes. En effet, la valeur de l'aire sous la courbe ROC ci-dessous est de $84,19 \%$. 
Graphique 2: Courbe ROC donnant le pouvoir discriminatif du modèle saturé.

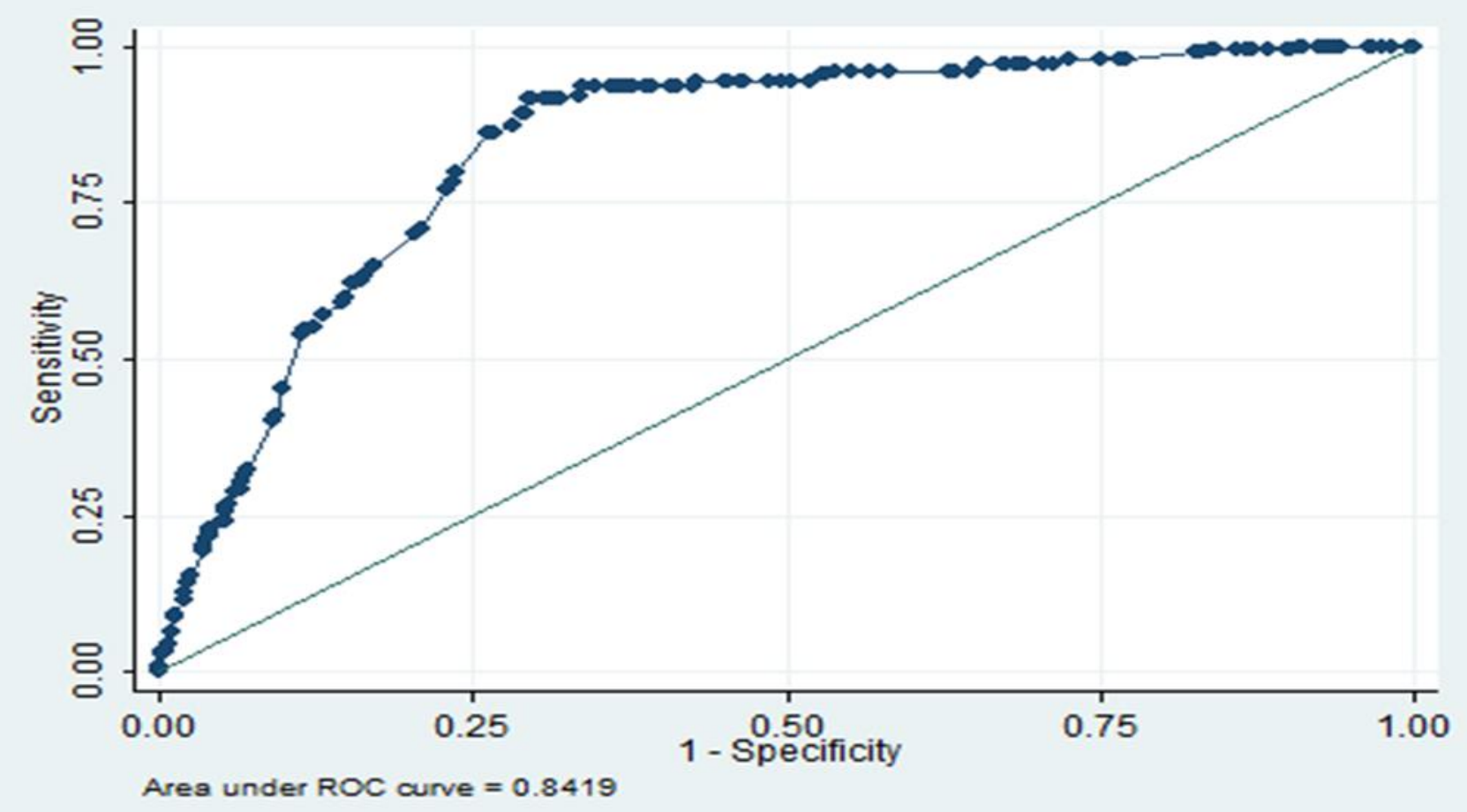




\subsubsection{Essai d'explication}

L'examen des effets bruts de chaque variable indépendante sur la variable dépendante consignés dans le tableau 1 ci-après permet de constater que le statut de la femme a un effet brut significatif sur l'émigration internationale des femmes. Son influence persiste même après le contrôle de toutes les autres variables prises en compte dans l'étude. Le statut de la femme constitue ainsi un facteur explicatif important des comportements migratoires des femmes. En effet, toutes choses égales par ailleurs, les femmes ayant un statut élevé ont 27,6\% moins de chances d'effectuer ou d'avoir l'intention d'effectuer une émigration internationale que celles ayant un statut faible. Ce résultat est en accord avec celui de l'analyse descriptive.

Pour contrôler le contexte dans lequel ces femmes vivent, il a été introduit, successivement, dans le modèle la région et le milieu de résidence (M3). Après ce contrôle, le statut de la femme maintient son effet significatif sur la variable dépendante. Autrement dit, quel que soit le contexte dans lequel les femmes vivent, le statut demeure un facteur de différenciation dans la pratique migratoire. Cependant, on notera que le contrôle de ces deux variables diminue les chances des femmes qui ont un statut élevé de connaitre l'évènement comparées à celles qui ont un statut faible. En effet, après contrôle les femmes de statut élevé ont $30 \%$ fois moins de chance d'effectuer ou d'avoir l'intention d'effectuer une émigration internationale (au lieu de $27,6 \%$ fois moins au niveau brut). Les comportements différentiels des femmes s'expliquent donc, dans une moindre mesure, par les différences de contexte de résidence de ces dernières. Les femmes résidant dans la région Soudano-Sahélienne n'ont pas les mêmes chances d'émigrer ou d'avoir l'intention d'émigrer que celles résidant dans les régions Nord-Soudanienne et Sud-Soudanienne. Cette inégalité des chances pourrait être attribuable à l'environnement économique, climatique et culturel spécifique à chaque région.

Au niveau du modèle M4 qui prend en compte le sexe du chef de ménage en plus des variables contextuelles, on observe que les chances d'émigrer ou d'avoir l'intention d'émigrer des femmes de statut élevé ont diminué considérablement puisqu'elles ont maintenant $37,8 \%$ moins de chance d'émigrer que celles de statut faible. Aussi, la significativité de l'influence du statut de la femme est passé du seuil de $5 \%$ au seuil de $1 \%$. Les femmes vivant dans des ménages dirigés par les femmes ont une plus grande propension à émigrer ou d'avoir l'intention d'émigrer (2,55 fois plus de chance). Cette variable inhibe l'effet du statut de la femme sur sa propension à émigrer ou son intention, c'est-à-dire l'écart par rapport aux comportements migratoires des femmes s'amoindrirait si elles résidaient toutes dans des ménages dirigés uniquement par des hommes ou uniquement par des femmes. 
Le modèle M5 dans lequel est intégré la taille du ménage se traduit par une augmentation de l'odds ratio associé aux femmes de statut élevé. Il est passé de 0,622 à 0,633 . Autrement dit, après le contrôle par la taille du ménage, la chance de connaitre l'évènement augmente chez les femmes de statut élevé. Cette augmentation est cependant très insignifiante. Toute chose qui laisse émettre la réserve quant au caractère médiatique de la taille du ménage dans le processus migratoire. En plus le statut de la femme est resté significatif au seuil de 1\% de M4 à M6. Néanmoins on remarque d'une manière générale que le modèle gagne en performance puisqu'au fur et à mesure qu'on contrôle des variables, le modèle explique mieux le phénomène étudié.

L'introduction de la variable niveau de vie au modèle M6 montre que les chances des femmes de statut élevé diminuent. Elles ont 37,4\% moins de chances de connaitre ou d'avoir l'intention de connaitre l'évènement que leurs homologues de statut social faible contre $36,7 \%$ dans le modèle M5. Tout comme dans le cas de la taille du ménage, après le contrôle par le niveau de vie du ménage, la variation de l'odd ratio est très modique pour qu'on puisse justifier le caractère médiatique de cette variable vis-à-vis de l'influence du statut de la femme sur l'émigration internationale.

Considérant le modèle complet $\mathrm{M} 7$, on note qu'après l'ajout de la variable expérience migratoire du ménage, la significativité du statut de la femme a diminué. Elle est passée du seuil de 1\% au seuil de 5\%. L'expérience migratoire est donc une variable médiatrice. Concrètement dit, le statut de la femme influence l'émigration internationale ou les intentions migratoires mais cette influence reste conditionnée plus par l'expérience que les ménages ont de la migration internationale. On peut dire encore que si tous les ménages dans lesquels vivent les femmes ont les mêmes expériences en matière de migrations internationales alors les femmes, quel que soit leurs statuts, tendraient à avoir les mêmes comportements migratoires.

Par ailleurs, ont également un effet net significatif au seuil de 5\% sur le statut migratoire, la région de résidence, le milieu de résidence, le sexe du chef de ménage, la taille du ménage et l'expérience migratoire du ménage. Ce qui signifie que non seulement ces variables contribuent à renforcer l'effet du statut de la femme sur l'émigration internationale féminine mais elles constituent également des variables explicatives importantes, exerçant des effets propres sur le phénomène en question. On peut souligner que les femmes, indépendamment de leurs statuts, résidant dans la région NordSoudanienne ont plus de chance d'émigrer ou d'avoir l'intention d'émigrer que celles de la région Sud-Soudanienne. Aussi, les femmes résidant dans les ménages dont le chef est une femme ont deux fois plus de chance d'émigrer ou d'avoir l'intention que celles résidant dans des ménages dont le chef est un homme. De même, les femmes habitant dans des ménages ayant une expérience migratoire ont 28,62 fois plus de chance d'effectuer ou d'avoir 
l'intention d'effectuer une émigration internationale que celles habitant dans des ménages n'ayant aucune expérience migratoire. Enfin, concernant la taille du ménage, les femmes appartenant aux ménages ayant moins de sept membres ont 1,53 fois plus de chances de partir ou d'avoir l'intention de partir à l'étranger que celles vivant dans des ménages ayant plus de six membres. Seul le niveau de vie du ménage n'a pas d'effet net significatif sur le statut migratoire, au seuil de 5\%. Bref, le statut de la femme est loin d'être le seul facteur explicatif de l'émigration internationale féminine.

Tableau 1 : Rapport de chance d'émigrer ou d'avoir l'intention d'émigrer à l'internationale chez les femmes

\begin{tabular}{|c|c|c|c|c|c|c|c|c|}
\hline \multirow{3}{*}{$\begin{array}{c}\text { Variables } \\
\text { indépendantes }\end{array}$} & \multicolumn{8}{|c|}{ Rapport de chances } \\
\hline & \multirow{2}{*}{$\begin{array}{c}\begin{array}{c}\text { Effets } \\
\text { bruts }\end{array} \\
\text { M0 }\end{array}$} & \multicolumn{7}{|c|}{ Effets nets } \\
\hline & & M1 & M2 & M3 & M4 & M5 & M6 & M7 \\
\hline \multicolumn{8}{|c|}{ Statut de la femme } & $* *$ \\
\hline Statut faible & Réf. & Réf. & Réf. & Réf. & Réf. & Réf. & Réf. & Réf. \\
\hline Statut élevé & $0.724^{* *}$ & $0.724^{* *}$ & $0.697^{* *}$ & $0.700^{* *}$ & $0.622^{* * * *}$ & $0.633^{* * *}$ & $0.626^{* * *}$ & $0.723^{* *}$ \\
\hline \multicolumn{8}{|c|}{ Région de résidence } & $* * * *$ \\
\hline $\begin{array}{l}\text { Soudano- } \\
\text { sahélienne }\end{array}$ & $0.435^{* * *}$ & & $0.423^{* * *}$ & $0.422^{* * * *}$ & $0.423^{* * *}$ & $0.433^{* * *}$ & $0.429^{* * *}$ & $0.466^{* * *}$ \\
\hline $\begin{array}{c}\text { Nord- } \\
\text { Soudanienne }\end{array}$ & Réf & & Réf & Réf & Réf & Réf & Réf & Réf \\
\hline $\begin{array}{c}\text { Sud- } \\
\text { Soudanienne }\end{array}$ & $0.798^{\mathrm{ns}}$ & & $0.771^{\mathrm{ns}}$ & $0.772^{\mathrm{ns}}$ & $0.750^{\text {ns }}$ & $0.755^{\mathrm{ns}}$ & $0.787^{\mathrm{ns}}$ & $1.043^{\text {ns }}$ \\
\hline \multicolumn{8}{|c|}{ Milieu de résidence } & *** \\
\hline Urbain & Réf & & & Réf & Réf & Réf & Réf & Réf \\
\hline Rural & $1.025^{\mathrm{ns}}$ & & & $1.017^{\mathrm{ns}}$ & $1.122^{\mathrm{ns}}$ & $1.192^{\mathrm{ns}}$ & $1.142^{\mathrm{ns}}$ & $0.678^{* *}$ \\
\hline \multicolumn{8}{|c|}{ Sexe du chef du ménage } & $* * *$ \\
\hline Masculin & Réf & & & & Réf & Réf & Réf & Réf \\
\hline Féminin & $2.221^{* * *}$ & & & & $2.551^{* * *}$ & $2.363^{* * *}$ & $2.484^{* * *}$ & $2.059^{* * *}$ \\
\hline \multicolumn{8}{|c|}{ Taille du ménage } & ** \\
\hline $\begin{array}{l}\text { Moins de sept } \\
\text { personnes }\end{array}$ & $1.617^{* *}$ & & & & & $1.455^{* *}$ & $1.379^{*}$ & $1.537^{* *}$ \\
\hline $\begin{array}{l}\text { Sept personnes } \\
\text { et plus }\end{array}$ & Réf & & & & & Réf & Réf & Réf \\
\hline \multicolumn{9}{|c|}{ Niveau de vie du ménage } \\
\hline Niveau faible & $1.166^{\mathrm{ns}}$ & & & & & & $1.068^{\mathrm{ns}}$ & $1.126^{\mathrm{ns}}$ \\
\hline Niveau élevé & Réf & & & & & & Réf & Réf \\
\hline \multicolumn{8}{|c|}{ Expérience migratoire du ménage } & $* * *$ \\
\hline $\begin{array}{c}\text { Avec } \\
\text { expérience }\end{array}$ & $24.533^{* * * *}$ & & & & & & & $28.625^{* * *}$ \\
\hline Sans expérience & Réf & & & & & & & Réf \\
\hline \multicolumn{2}{|l|}{ Chi 2} & 4,41 & 19,38 & 19,39 & 39,87 & 45,48 & 45,28 & 292,65 \\
\hline
\end{tabular}

Source : Exploitation des données de l'enquête IPPMD au Burkina Faso 
En somme, l'hypothèse (H1) selon laquelle les femmes de statut élevé ont plus de chance d'émigrer ou d'avoir l'intention d'émigrer à l'internationale que celles ayant un statut faible est infirmée. En effet, au modèle complet M7, elles ont $27,7 \%$ moins de chance que celles de statut faible.

A la lumière du tableau ci-dessus, on relève que, les femmes qui résident dans la région Soudano-Sahélienne ont moins de chance d'émigrer ou d'avoir l'intention d'émigrer que celles qui résident dans la région NordSoudanienne. Cette différence est significative au seuil de $1 \%$. Cela signifie que la différence observée entre les deux régions au modèle global n'est pas probablement relative au statut de la femme mais plutôt aux régions ellesmêmes et que la région de résidence est une variable explicative de l'émigration internationale ou des intentions d'émigrer des femmes. Aussi, le milieu de résidence des femmes a un effet net significatif dans le modèle global. Cependant, lorsqu'on contrôle par le statut de la femme, il perd toute significativité au seuil de 5\%. Autrement dit, dans un groupe de femmes de même statut, il n'y a pas de différence de comportement face à l'émigration internationale ou aux intentions d'émigrer des femmes à l'international. Les femmes de statut élevé résidant en milieu urbain auraient les mêmes comportements que celles résidant en milieu rural. Il en est de même chez les femmes de statut faible. Ce résultat est en accord avec celui obtenu au niveau descriptif en ce qui concerne les femmes résidant en milieu urbain. On peut donc dire que, les femmes n'émigrent pas ou n'ont pas des intentions d'émigrer parce qu'elles résident en milieu rural ou urbain mais plutôt parce qu'elles ont un statut faible, c'est-à-dire, la différence observée au niveau global entre le milieu urbain et le milieu rural chez toutes les femmes sans distinction de statut, est relative à la différence de statut qu'au milieu luimême. Par conséquent, l'hypothèse (H2) qui suppose que, la région et le milieu de résidence ont, chacune, en plus de l'effet "net » direct, un effet indirect sur le statut d'émigration international via le statut de la femme est vérifié.

En plus du statut de la femme, on retiendra également que l'expérience migratoire des ménages dans lesquels vivent les femmes constitue un facteur décisif voire primordial dans l'explication de leurs mouvements migratoires entre le Burkina Faso et le reste du monde. Le sexe du chef du ménage et la taille du ménage constituent des variables de différenciation à la pratique migratoire des femmes. Elles ont chacune un effet «net » direct mais aussi de façon indirect à travers le statut de la femme. Le niveau de vie du ménage n'influence pas la posture des femmes en ce qui concerne le fait migratoire. Donc, l'hypothèse (H3) qui stipule que, l'expérience migratoire et le niveau de vie du ménage ainsi le sexe du chef de ménage et la taille du ménage ont 
non seulement un effet «net » direct sur le statut d'émigration international mais aussi un effet indirect via le statut de la femme est partiellement vrai.

Cependant, la compréhension de ces résultats nécessite qu'on fasse recourt non seulement aux approches mobilisées mais également et surtout au contexte burkinabé dans lequel se trouvent les femmes au risque de «tomber dans l'erreur fatale de l'occidentalocentrisme » pour reprendre Perroux cité par Lututala (1995).

\section{Discussion}

Se référant aux résultats des analyses ci-dessus, le résultat selon lequel les femmes de statut faible ont plus de chance d'émigrer à l'international infirmant donc l'hypothèse $(\mathrm{H} 1)$, trouverait son explication dans du contexte burkinabé. D'abord, le pays partage près de $80 \%$ de ses flux migratoires avec la Côte d'Ivoire dont plus de $74,6 \%$ en ce qui concerne les émigrations féminines. Ensuite, le contexte environnemental défavorable du pays conjugué avec la pauvreté conduit les jeunes hommes burkinabés valides et d'origine rurale à émigrer vers le reste du monde notamment la Côte d'Ivoire et l'Italie à la recherche de meilleures conditions de vie. Et enfin, non seulement 68,6\% des femmes de statut faible résident en milieu rural mais également $62 \%$ des émigrantes parmi ces dernières résidaient aussi en milieu rural burkinabé selon les données de cette étude. Ces trois constats convergent pour expliquer la prédominance des femmes de statut faible dans les flux migratoires vers l'extérieur du pays. En effet, les motifs des émigrations féminines sont, en générale, les regroupements familiaux et dans le cas du Burkina Faso les liens familiaux entre les membres vivant à l'extérieur et ceux restés au pays sont préservés, le plus souvent, à travers le mariage. Par ailleurs, le Burkina Faso est membre de la CEDEAO qui est un espace de libre circulation des personnes donc un espace non discriminatoire en matière de migration. Autrement dit, le coût de l'émigration est réduit au coût net du déplacement (frais de transports) si l'on fait fi des autres coûts non matériels.

Sur le plan empirique, plusieurs auteurs ont abouti à des conclusions semblables dans d'autres contextes. Ainsi, Zourkaléini (2007) dans son étude sur les déterminants individuels et contextuels de l'émigration internationale au Burkina Faso avait souligné que, les femmes qui partent pour une autre destination que la Côte d'Ivoire sont les seules à afficher une différence significative au niveau de l'activité salariale. Autrement, les femmes à destination de la Côte d'Ivoire sont celles qui n'exercent pas d'activités rémunérées. Dans cette étude, les femmes n'exerçant pas d'activités rémunérées représentent $78,66 \%$ des femmes de statut faible (tableau Annexe) et $73,15 \%$ des femmes de statut faible ayant quitté le territoire national. Ce qui rejoint le résultat de Zourkaléini (2007). Les résultats de Kombieni (2015) au Togo et (Tacoli, 1999) au Philippines vont également dans le même sens. 
L'expérience migratoire du ménage dans lequel elles résident est déterminante dans l'explication de l'émigration internationale des femmes. Au Burkina Faso, l'expérience en matière de migration internationale qu'ont les ménages dans lesquels vivent les femmes est une conséquence directe des forts taux d'émigration. En effet, le solde migratoire du Burkina Faso était négatif lors des trois derniers recensements réalisés, le solde migratoire du recensement de 2019 n'étant pas disponible au moment de cet écrit. C'est ainsi que les femmes ${ }^{3}$ restées aux lieux d'origines des émigrants acquièrent donc de l'expérience migratoire. En plus de ce contexte, on peut recourir à la théorie des réseaux sociaux pour mieux comprendre l'importance de l'expérience migratoire dans le cas des migrations féminines africaines. Selon Vause (2012), cette théorie est capitale en raison des risques élevés que peuvent courir les femmes en migrations comparativement aux hommes. Les femmes, avant de quitter leurs ménages savent, le plus souvent, où aller, quand et comment. Empiriquement, Hazard B. (2007), cité par Zidnaba (2016) a montré que les migrations burkinabè en Italie se sont construites dans l'optique des réseaux. A partir des primo-arrivés dans les années 1980, les différentes vagues successives se sont appuyées sur les solidarités familiales ou communautaires de ces derniers à telle enseigne qu'en moins de trois décennies la communauté burkinabè d'Italie constitue l'une des plus importante hors d'Afrique. Les femmes sont également concernées par cette communauté.

La conclusion relative au caractère masculin ou féminin de la personne responsable du ménage (chef de ménage) dans l'explication du phénomène s'expliquerait dans les communautés burkinabés. En effet, les sociétés burkinabés sont à majorité patriarcales et gérontocratiques. La place de la femme est dans le ménage. Près de $90 \%$ des ménages sont dirigés par les hommes contre seulement à peine $10 \%$ de ménages dont le chef est une femme. Dans un tel contexte caractérisé par un pouvoir absolu détenu par les hommes qui ont, généralement, des attitudes défavorables à l'émigration internationale des femmes, on peut comprendre que les femmes, quel que soit leur statut, aient plus de chance d'émigrer si elles résident dans des ménages dont le contrôle est assuré par une femme. Au niveau des approches, l'approche genre peut permettre de mieux saisir ce résultat. Du reste, pour certains auteurs, Le Jeune et al. (2013) et Piché (2013), la migration des femmes s'inscrit donc dans des rapports de pouvoir et les recherches examinent non seulement la manière dont ces rapports façonnent les décisions migratoires des femmes, mais aussi comment la migration féminine peut changer le système patriarcal. Sur le plan empirique, Oso et Catarino (1997)

${ }^{3}$ La femme peut être, ici, la femme d'un migrant ou tout simplement une femme résidant dans le ménage d'origine d'un migrant. 
sont arrivés à la conclusion que les femmes deviennent chefs que dans certaines circonstances telles que le célibat, le divorce, le veuvage ou encore l'émigration des hommes qui les contraint à devenir chefs de ménages. Ces résultats, en ce qui concerne l'émigration et le célibat, confortent les propositions souvent formulées dans la littérature pour expliquer le comportement migratoire des femmes basé sur la théorie du regroupement familial.

Cependant, les limites des données n'ont pas permis d'établir des conclusions sur la relation statut de la femme et l'émigration internationale prenant en compte toutes les variables relatives aux différents concepts du cadre conceptuel élaboré. D'une part, une des limites est relative aux variables composites utilisées dans l'étude. En effet, le statut de la femme est avant tout social, à la fois multidimensionnel et complexe. Sa construction nécessite donc que soit pris en compte d'autres facteurs tels les caractéristiques du conjoint de la femme, la vie associative et politique de la femme, son accès aux moyens de production, les caractéristiques de ses enfants et parents, etc. qui sont susceptible d'influencer sa position dans la société. Or, de telles variables ne sont pas disponibles dans la base de données. D'autre part, la construction de l'indicateur « niveau de vie du ménage » à partir des biens et équipements du ménage présente également des biais. En effet, dans la mesure de l'aisance des 2200 ménages, cet indicateur n'est qu'approximatif car à moyens économiques et financiers égaux, les ménages n'investissent pas de la même manière dans les biens et équipements sur lesquels se basent les données d'enquêtes et de recensements pour construire l'indicateur de niveau de vie. Cet investissement dépend du contexte. Par exemple, la possession d'un réfrigérateur est plus tributaire de la disponibilité de l'électricité que celle des moyens financiers ou encore la possession d'une porte en métallique pour garder sa maison en sécurité dans certains villages est aussi révélateur de richesse que la possession d'un ordinateur en ville.

Une autre limite des données qui pourrait aussi être relevée est la disponibilité des données par rapport à la zone de départ seulement, ne capturant de ce fait que les intentions de migration ou l'expérience des migrantes de retour qui pourrait représenter une certaine catégorie de migrantes dont les caractéristiques sont différentes de celles des migrantes définitives et de longues durées dont des données depuis les zones d'accueil auraient permis de cerner les caractéristiques et le profil. Certes, cela requiert des ressources conséquentes, mais bon tout de même de relever cette limite et l'éventuel biais de sélection qui pourrait en découler. Entre autres suppositions, cela pourrait par exemple expliquer l'absence d'un effet statistiquement significatif en milieu urbain.

Un autre point de discussion pourrait s'articuler sur le développement du caractère passif et actif de la migration au regard de la relation négative 
entre le statut social et la propension à migrer. La relation ne devrait pas aussi être vue dans une perspective uniquement unilatérale. D'aucuns parleraient d'interrelations pour évoquer la réciprocité des deux phénomènes, car autant le statut social influencerait le statut migratoire, autant le statut migratoire pourrait affecter le statut social.

\section{Conclusion}

Des études ont mis en exergue l'influence des éléments constitutifs du statut de la femme sur la pratique migratoire, notamment le statut matrimonial de la femme, son niveau d'instruction, son statut d'activité, son lien de parenté avec le chef du ménage et son âge. C'est au regard de ces aspects parcellaires sur le sujet qui s'influencent mutuellement que ces cinq variables ont été regroupés dans un même indicateur qu'est le statut de la femme afin de saisir son influence sur l'émigration internationale chez les femmes âgées de quinze (15) ans et plus au Burkina Faso.

Avec une méthodologie alliant analyses bivariées (test de chi2), multivariées descriptive (AFCM) et explicative (régression logistique binomiale), il ressort que les objectifs ont été atteints. Un modèle à pas croissant a permis de saisir les mécanismes d'action du statut de la femme et de s'assurer de la validité des observations descriptives qui peuvent s'avérer somme toute fallacieuses, en appliquant la régression logistique binomiale. Cela a permis de confronter les hypothèses issues de la théorisation aux résultats empiriques. Il en résulte que le statut de la femme agit négativement sur la propension de la femme à émigrer ou à avoir l'intention d'émigrer. Les femmes de statut faible émigrent ou ont l'intention d'émigrer à l'international plus que celles de statut élevé. Ce résultat est d'autant plus vrai lorsqu'elles résident dans des ménages dirigés par des femmes. L'expérience migratoire du ménage constitue une possibilité pour l'accomplissement de l'acte migratoire chez ces femmes. Il ressort également que, la région et le milieu de résidence influencent directement l'émigration et indirectement via le statut de la femme. Des trois hypothèses formulées, l'une (H2) est totalement confirmée, l'autre $(\mathbf{H 1})$ est infirmée. La troisième hypothèse $(\mathbf{H 3})$ est partiellement confirmée.

$\mathrm{Au}$ plan scientifique, cette étude, bien qu'elle s'avère exploratoire demande une plus grande investigation dans le cadre de recherches futures afin de contribuer davantage à la compréhension plus large sur les migrations féminines au Burkina Faso. Au regard de ces résultats, l'amélioration des conditions de vie des femmes et la réalisation d'enquêtes de type biographiques, qualitatives et quantitatives plus approfondies et spécifiques aux femmes au Burkina Faso mais aussi dans les pays de destination des migrantes serait souhaitable afin d'en connaître plus sur les interrelations entre le statut de la femme, l'émigration, l'autonomisation et développement local. 


\section{References:}

1. Adepoju, A. (2002). Fostering free movement of persons in West Africa: Achievements, constraints, and prospects for intraregional migration. International migration, 40(2), 3-28.

2. Ambrosetti, E., Tattolo, G., Toader, A., Kateb, K., \& Diguet, D. (2008). Femmes, rapports de genre et dynamiques migratoires. Population, 63(4), 767-793.

3. Antoine, P., \& Sow, O. (2000). Rapport de genre et dynamiques migratoires. Rapports de genre et question de population, 112-123.

4. Assogba, M. L. (1989). Statut de la femme et fécondité dans le Golfe du Bénin : La décision de la fécondité pour le statut ou par le statut? [Thèse de Doctorat en démographie]. Université de Paris 1.

5. Assogba, M. L. (1990). Statut de la femme, structures familiales, fécondité : Transitions dans le Golfe du Bénin. Centre français sur la population et le développement.

6. Assogba, M. L. (1992). Statut de la femme et migration urbaine dans le Golfe du Bénin : De la décision à l'insertion. Cahiers québécois de démographie, 21(1), 121-149.

7. Ba, C. O. (1998). Dynamiques migratoires et changements sociaux au sein des relations de genre et des rapports jeunes/vieux des originaires de la moyenne vallée du fleuve sénégal [Thèse de Doctorat]. Université Cheikh Anta Diop (U.C.A.D.) de Dakar.

8. Beauchemin, C. (2011). Rural-urban migration in West Africa: Towards a reversal? Migration trends and economic situation in Burkina Faso and Côte d'Ivoire. Population, Space and Place, 17(1), 47-72.

9. Bisilliat, J. (1983). Le travail de la femme et ses conséquences sur la famille.

10. Bocquier, P., \& Traoré, S. (2000). Urbanisation et dynamique migratoire en Afrique de l'Ouest. La croissance urbaine en panne. http://hdl.handle.net/2078.1/78555.

11. Brachet, J. (2007). Un désert cosmopolite. Migrations de transit dans la région d'Agadez (Sahara nigérien) [Thèse de doctorat en géographie]. Université Panthéon-Sorbonne-Paris I.

12. Bréant, H. (2012). Démontrer le rôle positif des migrations internationales par les chiffres. Une analyse de la rhétorique institutionnelle du système des Nations unies. Mots. Les langages $d u$ politique, 3, 153-171.

13. Cambien, A. (2008). Une introduction à l'approche systémique: Appréhender la complexité. Certu.

14. Caselli, G., Vallin, J., \& Wunsch, G. (2003). Démographie: Analyse et synthèse. Les déterminants de la migration (Vol. 4). INED. 
15. Catarino, C., \& Morokvasic, M. (2005). Femmes, genre, migration et mobilités (Vol. 21). Université de Poitiers.

16. Chort, I., De Vreyer, P., Zuber, T., \& Guerrouche, K. (2020). Mobilité genrée au Sénégal. Population, 75(2), 297-323.

17. Dabire, B. (2004). Les determinants familiaux de l'emigration rurale au Burkina Faso. African Population Studies, 22(1), 108-135.

18. De Lange, A. (2007). Child labour migration and trafficking in rural Burkina Faso. International Migration, 45(2), 147-167.

19. Félix, Y., Lydie-Marcelle, K. A., Larissa, K. A., \& Alain, T. (2017). Socio-Anthropologie Du Processus D'autonomisation Des Femmes Rurales Ivoiriennes À Travers Les Activités Génératrices De Revenus. European Scientific Journal, ESJ, 13(32), 303-303. https://doi.org/10.19044/esj.2017.v13n32p303.

20. Findley, S. (1989). Les migrations feminines dans les villes africaines : Une revue de leurs motivations et experiences. L'insertion urbaine des migrants en Afrique, 55-70.

21. Gregory, J. W., Saint-Pierre, M.-H., \& Simmons, A. B. (1986). Structure démographique des ménages et comportement migratoire en Haute-Volta. Collection tirés à part/Université de Montréal, Dept. de démographie; no. 215, 111-141.

22. Henry, S., Boyle, P., \& Lambin, E. F. (2003). Modelling interprovincial migration in Burkina Faso, West Africa: The role of sociodemographic and environmental factors. Applied Geography, 23(2), 115-136.

23. INSD. (2009). Recensement général de la population et de l'habitat du Burkina Faso de 2006 (RGPH-2006), theme 8: Migrations (p. 150) [Rapport].

24. INSD et ICF International. (2012). Enquête Démographique et de Santé et à Indicateurs Multiples (EDSBF-MICS IV) (p. 501) [Rapport].

25. Kamdem, K. H. (2006). Genre et fécondité au Cameroun: Etude comparative des Bamiléké et des Bëti [Thèse de Doctorat en démographie]. Université de Yaoundé II Soa, IFORD.

26. Kamgno, H. (1993). Rôle, statut de la femme et SIDA : cas du village Mindourou à l'EST-Cameroun [DED]. Université de Yaoundé II Soa, IFORD.

27. Kenne, A. R. W. (2011). Emigration internationale féminine d'origine camerounaise: Rechereche des facteurs explicatifs [Mémoire de Master en Démographie]. Université de Yaoundé II Soa, IFORD.

28. Kobiané, J.-F. (2007). Ethnies, genre et scolarisation au Burkina Faso : $\mathrm{Du}$ discours anthropologique aux résultats statistiques. Thérèse 
LOCOH (éd.), Genre et sociétés en Afrique. Implications pour le développement. Collection, Les cahiers de l'INED, 160, 221-241.

29. Kombieni, H. (2015). Migrations et femmes au Bénin: Analyse de quelques déterminants. Sciences Humaines, 1 .

30. Le Jeune, Gaël. (2007). Migration et insertion des femmes dans le marché du travail urbain: Une remise en question des rapports de genre ? Dynamique migratoire, insertion urbaine et environnement au Burkina Faso : Au-delà de la houe, Paris, L'Harmattan, 189-212.

31. Le Jeune, Gael, Piché, V., \& Poirier, J. (2013). L'émergence d'une migration féminine autonome du milieu rural vers le milieu urbain au Burkina Faso ? African Population Studies, 20(2), 102-123.

32. Lee, E. S. (1966). A theory of migration. Demography, 3(1), 47-57.

33. Lesclingand, M., \& Hertrich, V. (2017). Quand les filles donnent le ton. Migrations adolescentes au Mali. Population, 72(1), 63-93. https://doi.org/10.3917/popu.1701.0063.

34. Lututala, M. B. (1995). Les migrations africaines dans le contexte socio-économiques actuel : Une révue critique des modèles explicatifs. In La Sociologie des populations (p. 391-416). PUM/AUPELF-UREF.

35. Lutz, H. (2010). Gender in the migratory process. Journal of ethnic and migration studies, 36(10), 1647-1663.

36. Mankou, B. A. (2011). Cybermigration maritale des femmes camerounaises de Yaoundé vers le NORD-PAS-DE-CALAIS : analyse sociologique et enjeux sociaux d'une migration nouvelle [Thèse de Doctorat en Sociologie-Option changement social]. Université de Lille 1.

37. Marsicano, E., Lydié, N., \& Bajos, N. (2011). Genre et migration : L'entrée dans la sexualité des migrants d'Afrique subsaharienne en France. Population, 66(2), 313-341.

38. Mimche, H., Nganawara, D., \& Ouédraogo, H. (2013). Migrations féminines et histoire africaine : Pratiques d'hier, réalité d'aujourd'hui. Afrika Zamani, 18-19, 161-179.

39. Mimche, H., Yambéné, H., \& ZOA ZOA, J. (2005). La féminisation des migrations clandestines en Afrique noire. colloque: Mobilité au féminin, 54-77.

40. Ministère de la promotion de la femme. (2009). Document de la politique nationale genre du Burkina Faso (p. 56).

41. Ministère de l'économie, des finances et du développement. (2017). Stratégie Nationale de Migration 2016-2025 (p. 47) [Document de travail].

42. Miranda, A., Ouali, N., \& Kergoat, D. (2011). Les mobilisations des migrantes: Un processus d'émancipation invisible? Cahiers $d u$ Genre, 51, 5-24. https://doi.org/10.3917/cdge.051.0005. 
43. Morokvasič, M. (1986). Émigration des femmes : Suivre, fuir ou lutter. Nouvelles questions féministes, 65-75.

44. Moujoud, N. (2008). Effets de la migration sur les femmes et sur les rapports sociaux de sexe: Au-delà des visions binaires. Cahiers $d u$ CEDREF, 57-79.

45. Néya, S. (2016). Les mobilités spatiales féminines entre logiques individuelle et familiale. L'exemple des migrantes burkinabè entre le Burkina Faso et la Côte d'Ivoire. EchoGéo, 37.

46. Nganawara, D. (2017). Analyse de la migration. IFORD.

47. OIM. (2016). Migration au Burkina Faso : Profil migratoire 2016 (p. 97). www.iom.int.

48. Omeyaka, B. L. S. L. (2018). Lutte De Leadership Feminin Au Pouvoir Et $(\mathrm{Re})$ Structuration De Rapports Sociaux De Sexe En Province Orientale. European Scientific Journal, ESJ, 14(35), 84-84. https://doi.org/10.19044/esj.2018.v14n35p84.

49. Oppong, C. (1980). A synopsis of seven roles and status of women: An outline of a conceptual and methodological approach. World Employment Programme Research, Population and Labour Policies Programme Working Papers, 94, ii, 55 p. POPLINE.

50. Oso, L., \& Catarino, C. (1997). Femmes chefs de ménage et migration. Femmes du Sud et chefs de famille, 63.

51. Ouedraogo, D. (2007). Au-delà de la houe: La mobilité géographique comme déterminant majeur de la mobilité sociale ascendante au Burkina Faso. Dynamique migratoire, insertion urbaine et environnement au Burkina Faso. Au-delà de la houe, 265-283.

52. Pessar, P. R. (1999). The role of gender, households, and social networks in the migration process: A review and appraisal. na.

53. Piché, V. (2013). Les théories de la migration (Vol. 1). Ined.

54. Rakotondrabe, F. P. (2004). Statut de la femme, prise de décision et santé des enfants à Madagascar [Thèse de Doctorat en démographie]. Université de Yaoundé II Soa, IFORD.

55. Rouamba, P. I. L. (2011). La participation des femmes à la vie politique au Burkina (1957-2009) [PhD Thesis]. Université du Québec à Montréal.

56. Roulleau-Berger, L. (2010). Migrer au féminin. Presses universitaires de France.

57. Rwenge, J.-R. M. (2003). Statut de la Femme et Utilisation des Condoms au Cameroun. African Journal of Reproductive Health, 74-88.

58. Rwenge, J.-R. M. (2007). Statut de la femme, planification familiale et fecondite a Mbalmayo et Bafoussam, Cameroun. African Population Studies, 22(1), 85. 
59. Rwenge, M. (1995). Statut de la femme, comportements sexuels et SIDA en Afrique subsaharienne : Le cas du Cameroun. Séminaire sur les aspects socio-économiques et démographiques du HIV/SIDA en Afrique, Abidjan, 25-28.

60. Sakho, P., \& Dial, F. B. (2010). Migration clandestine féminine. Etude de cas de Dakar et sa banlieue.

61. Sarr, F., Ndiaye, L., Wade, A., Sow, A., \& Kane, M. S. L. (2009). Genre et migration clandestine dans les zones côtières au Sénégal. Synthèse des résultats préliminaires (Mai 2009)Dakar: Laboratoire Genre IFAN.

62. Sigrun, H. (2004). Profil genre du Burkina Faso : Vers l'égalité entre les genre au Burkina faso ( ${ }^{\circ}$ SIDA3965fr; p. 91). Asdi.

63. Tabutin, D., \& Schoumaker, B. (2020). La démographie de l'Afrique subsaharienne au XXIe siècle. Population, 75(2), 169-295.

64. Tacoli, C. (1999). International migration and the restructuring of gender asymmetries: Continuity and change among Filipino labor migrants in Rome. International migration review, 33(3), 658-682.

65. Tall, S. M., \& Tandian, A. (2010). Entre regroupement familial et migrations autonomes des femmes sénégalaises. Quelle analyse de genre des migrations sénégalaises?

66. Toma, S. (2014). L'influence mitigée des migrations masculines sur les activités économiques des femmes «qui restent»: Étude de cas dans la vallée du fleuve Sénégal. Cahiers québécois de démographie, 43(2), 345-374.

67. Vallin, J. (1988). Conférence sur le statut de la femme et l'évolution démographique dans le cadre du développement. Population (french edition), 1162-1169.

68. Vause, S. (2009). Genre et migrations internationales Sud-Nord: Une synthèse de la littérature. Département des sciences de la population et du développement, Université catholique de Louvain.

69. Vause, S. (2012). Différence de genre et rôles des réseaux migratoires dans la mobilité internationale des Congolais (RDC): Étude des tendances, des déterminants et des conséquences de la migration [Thèse de Doctorat en démographie]. Université Catholique de Louvain.

70. Vause, S., Toma, S., \& Richou, C. (2015). Peut-on parler de féminisation des flux migratoires du Sénégal et de la République démocratique du Congo? Population, 70(1), 41-67.

71. Zidnaba, I. (2016). Migrations internationales et développement: L'impact socio-économique des pratiques transnationales des émigrés de Béguédo résidant en Italie [Thèse de Doctorat en géographie, Université Ouaga I Pr Joseph KI-ZERBO]. 
http://www.burkinadiaspora.org/images/memoires/Th\%C3\%A8se $\% 2$ 0Zidnaba.pdf.

\section{Annexe}

Annexe 1 : Association entre statut de la femme et statut d'émigration

\begin{tabular}{|c|c|c|c|c|c|c|}
\hline \multirow{3}{*}{$\begin{array}{l}\text { Statut de la } \\
\text { femme }\end{array}$} & \multicolumn{4}{|c|}{ Statut d'émigration } & \multirow{3}{*}{ Total } & \multirow[b]{3}{*}{$\begin{array}{l}\text { Signification } \\
(\mathrm{Chi} 2)\end{array}$} \\
\hline & \multicolumn{2}{|c|}{ Migrante } & \multicolumn{2}{|c|}{ Non migrante } & & \\
\hline & $\begin{array}{c}\text { proportion } \\
(\%)\end{array}$ & effectifs & $\begin{array}{c}\text { proportion } \\
(\%)\end{array}$ & effectifs & & \\
\hline Statut faible & 5,34 & 108 & 94,66 & 1913 & 2021 & \\
\hline Statut élevé & 3,93 & 73 & 96,07 & 1786 & 1859 & \\
\hline Total & 4,66 & 181 & 95,34 & 3699 & 3880 & $* * *$ \\
\hline
\end{tabular}

Source : Exploitation des données de l'enquête IPPMD au Burkina Faso 
Annexe 2 : Proportion des femmes selon le statut de la femme et certaines caractéristiques individuelles

\begin{tabular}{|c|c|c|c|c|c|c|c|}
\hline \multirow[b]{2}{*}{ Variables } & \multirow[b]{2}{*}{ Modalités } & \multicolumn{2}{|c|}{ Statut faible } & \multicolumn{2}{|c|}{ Statut élevé } & \multicolumn{2}{|c|}{ Ensemble } \\
\hline & & Effectifs & $\begin{array}{c}\text { Proportion } \\
(\%)\end{array}$ & Effectifs & $\begin{array}{c}\text { Proportion } \\
(\%)\end{array}$ & Effectifs & $\operatorname{Proportion}(\%)$ \\
\hline \multirow{4}{*}{ Age } & $15-24$ ans & 772 & 58,31 & 552 & 41,69 & 1324 & 100 \\
\hline & $25-34$ ans & 738 & 68,27 & 343 & 31,73 & 1081 & 100 \\
\hline & Plus de 35 ans & 511 & 34,64 & 964 & 65,36 & 1475 & 100 \\
\hline & Total & 2021 & 52,09 & 1859 & 47,91 & 3880 & 100 \\
\hline \multirow{4}{*}{ Statut matrimoniale } & Mariée ou en union libre & 1606 & 64,58 & 881 & 35,42 & 2487 & 100 \\
\hline & Célibataires & 408 & 37,78 & 672 & 62,22 & 1080 & 100 \\
\hline & Divorcée/séparée/Veuve & 7 & 2,24 & 306 & 97,76 & 313 & 100 \\
\hline & Total & 2021 & 52,09 & 1859 & 47,91 & 3880 & 100 \\
\hline \multirow{5}{*}{ Statut d'activité } & Activité rémunérée & 69 & 29,87 & 162 & 70,13 & 231 & 100 \\
\hline & Activité non rémunérée & 1283 & 78,66 & 348 & 21,34 & 1631 & 100 \\
\hline & Inactive scolarisé & 68 & 12,12 & 493 & 87,88 & 561 & 100 \\
\hline & inactive non scolarisé & 601 & 41,25 & 856 & 58,75 & 1457 & 100 \\
\hline & Total & 2021 & 52,09 & 1859 & 47,91 & 3880 & 100 \\
\hline \multirow{4}{*}{ Niveau d'instruction } & Aucun & 1742 & 71,19 & 705 & 28,81 & 2447 & 100 \\
\hline & Primaire & 263 & 49,07 & 273 & 50,93 & 536 & 100 \\
\hline & Secondaire et plus & 16 & 1,78 & 881 & 98,22 & 897 & 100 \\
\hline & Total & 2021 & 52,09 & 1859 & 47,91 & 3880 & 100 \\
\hline \multirow{4}{*}{ Lien de parenté avec le CM } & Chef de ménage & 51 & 23,83 & 163 & 76,17 & 214 & 100 \\
\hline & Epouse/fille & 1795 & 58,64 & 1266 & 41,36 & 3061 & 100 \\
\hline & Autre lien & 175 & 28,93 & 430 & 71,07 & 605 & 100 \\
\hline & Total & 2021 & 52,09 & 1859 & 47,91 & 3880 & 100 \\
\hline
\end{tabular}

Source : Exploitation des données de l'enquête IPPMD au Burkina Faso 